\title{
MiRNA-132/212 regulates tight junction stabilization in blood-brain barrier after stroke
}

\author{
Haomin Yan (D) ${ }^{1}$, Hideaki Kanki ${ }^{1}$, Shigenobu Matsumura ${ }^{2}$, Tomohiro Kawano ${ }^{1}$, Kumiko Nishiyama ${ }^{1}$, Shintaro Sugiyama ${ }^{1}$, \\ Hiroshi Takemori ${ }^{3}$, Hideki Mochizuki ${ }^{1}$ and Tsutomu Sasaki ${ }^{1{ }^{\circledR}}$
}

(c) The Author(s) 2021

MicroRNA-132/212 has been supposed as a critical gene related to the blood-brain barrier (BBB) protection after stroke, but its regulation pathway including the upstream regulator and downstream targets is still unclear. Herein, we demonstrated the cAMP response element-binding protein (CREB)-regulated transcription coactivator-1 (CRTC1) to be the upstream regulator of miRNA132/212 using CRTC1 knockout and wild-type mice. CRTC1 deletion led to the reduction of miRNA-132/212 expression in mice brain after ischemic stroke, significantly increased infarct volume, and aggravated BBB permeability with worsening neurological deficits. Furthermore, we identified that miRNA-132 repressed Claudin-1, tight junction-associated protein-1 (TJAP-1), and RNA-binding Fox1 (RBFox-1) by directly binding to their respective 3 -untranslated regions, which alleviated the ischemic damage by enhancing neuronal survival and BBB integrity. Moreover, the co-culture of endothelial cells with CRTC1-deficient neurons aggravated the cell vulnerability to hypoxia, also supporting the idea that miRNA-132/212 cluster is regulated by CRTC1 and acts as a crucial role in the mitigation of ischemic damage. This work is a step forward for understanding the role of miRNA-132/212 in neurovascular interaction and may be helpful for potential gene therapy of ischemic stroke.

Cell Death Discovery (2021)7:380; https://doi.org/10.1038/s41420-021-00773-w

\section{INTRODUCTION}

Stroke is a leading cause of mortality and long-term disability worldwide [1, 2]. Although intensive efforts have been made to develop new pharmacological therapies, tissue plasminogen activator is still the only Food and Drug Administrationapproved medication for ischemic stroke, but its use is restricted to a narrow time window, with a high risk of hemorrhage [3]. Therefore, a new treatment approach with an extended therapeutic time window is urgently required for ischemic stroke.

MicroRNAs (miRNAs), a group of endogenous non-coding RNAs composed of $\sim 20$ nucleotides, function as a critical role in posttranscriptional gene regulation via sequence-specifically binding to the $3^{\prime}$-untranslated region (UTR) of one or more messenger RNAs (mRNAs) [4]. Previous studies have demonstrated that miRNA conservation is often cell-type and tissue-specific [5], implying that miRNAs play a functional role in intercellular interactions.

MiR-132/212 is a highly brain-enriched miRNA cluster and has been well studied as an upstream inhibitory regulator on mRNA expression in CNS [6]. Zuo et al. have recently reported that miR132 attenuates cerebral injury via repressing MMP9 [7]. Otherwise, increasing studies suggested miR-132/212 to be a downstream regulator mediated by CAMP response element-binding protein (CREB) in visual plasticity $[8]$, memory $[9,10]$, and pain regulation [11]. Our previous studies demonstrated that CREB-dependent gene transcription is mainly dependent on CREB-regulated transcription coactivator-1 (CRTC1) coactivation [12], and CRTC1-CREB complex exerts a neuroprotective effect by regulating several factors, including proliferator-activated receptor gamma coactivator-1 alpha (PGC-1a) $[13,14]$ and brain-derived neurotrophic factor (BDNF) [15]. Also, miR-132 is demonstrated to be regulated by CRTC1 [16]. Inspired by these studies, we hypothesized that the CRTC1 may be an upstream regulator of miRNA-132/212.

The disruption of blood-brain barrier (BBB) was one of the most common pathological events in the very acute stage of ischemic stroke $[17,18]$. As a highly dynamic and complex structure, BBB is composed of pericytes, astrocytes, endothelial cells, and basement membrane, and regulates the movement of substances between CNS and blood $[19,20]$. It is known that the BBB function is regulated by certain tight junction protein. Claudin-5, as the most highly expressed tight junction protein at BBB, its dysfunction is closely associated with ischemic stroke pathology [21]. BBB permeability was found to be enhanced in Claudin-5 deficient mice, as under physiological condition, the $443 \mathrm{Da}$ tracer leaked from the blood vessel to the parenchymal side of brain, but the $1.9 \mathrm{kDa}$ tracer did not leak [22]. Recently, Sladojevic et al. reported Claudin-1 accumulated in leaky brain microvessels after stroke, and Claudin-1 specifically blockage had beneficial effects on BBB permeability [23]. These studies demonstrated the crucial role of tight junction proteins like Claudins in BBB integrity maintenance, but their upstream mechanism in stroke remains unknown.

\footnotetext{
${ }^{1}$ Department of Neurology, Graduate School of Medicine, Osaka University, Yamadaoka 2-2, Suita, Osaka 565-0871, Japan. ${ }^{2}$ Laboratory of Nutrition Chemistry, Division of Food Science and Biotechnology, Graduate School of Agriculture, Kyoto University, Kyoto, Japan. ${ }^{3}$ Department of Chemistry and Biomolecular Science, Faculty of Engineering, Gifu University, Gifu, Japan. ${ }^{\bowtie}$ email: sasaki@neurol.med.osaka-u.ac.jp
} 

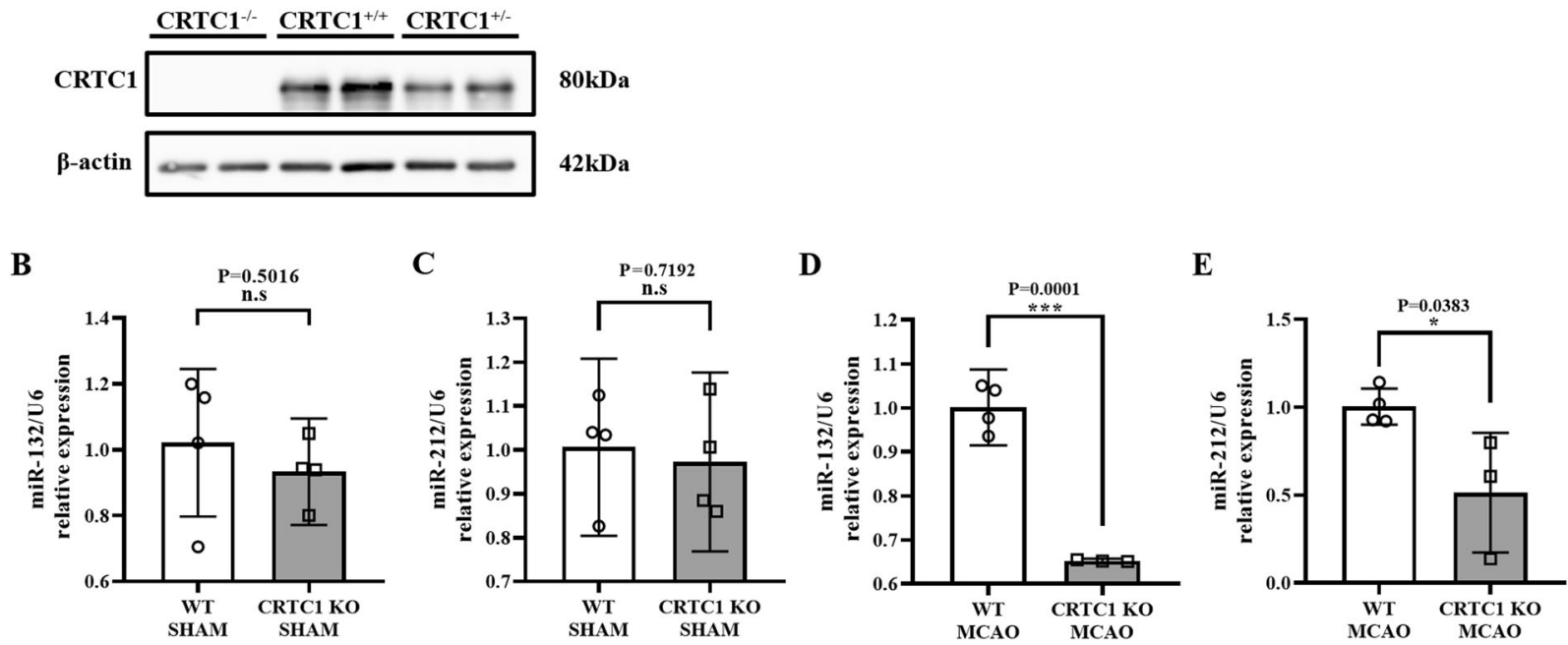

Fig. 1 CRTC1 deficiency induces miR-132/212 degradation after MCAO. A Western Blot shows CRTC1 has been completely deleted on C57B/ 6 background mouse. Quantitative polymerase chain reaction (qPCR) results of miR-132 (B) and miR-212 (C) in CRTC1 KO and WT mice brain under sham group. qPCR results of miR-132 (D) and miR-212 (E) in penumbra regions of CRTC1 KO and WT mice brain $24 \mathrm{~h}$ after MCAO. $n=$ 3-4 for each group.

To reveal the CRTC1-miR-132/212 signaling in the acute ischemic stroke phase, in this work we generated and subjected CRTC1 knockout and wild-type mice to $60 \mathrm{~min}$ transient middle cerebral artery occlusion (MCAO). Our findings showed that CRTC1 deficiency decreased miR-132/212 expression and aggravated mice neurological outcome after ischemic stroke. Furthermore, we identified and validated Claudin-1, tight junction-associated protein-1 (TJAP-1), and RNA-binding Fox-1 (RBFox-1) as targets of miR-132/212, which play a crucial role in the homeostasis of neuron-endothelial interaction under hypoxia. Besides, the in vitro experiments also suggested that CRTC1-mediated regulation of miR-132/212 on neuron-endothelial interaction act as a crucial role in the mitigation of cell susceptibility to hypoxia. This work offers new clues for understanding the role of miRNA-132/ 212 in neurovascular regulation and may provide a reference for potential novel therapy by controlling tight junction complex such as Claudin-1 and TJAP-1 after ischemic stroke.

\section{RESULTS}

CRTC1 deficiency suppresses the upregulation of miR-132/212 after cerebral ischemia in mice

To investigate the changes in miR-132/212 in ischemia, we induced MCAO in C57BL/6 mice. qPCR data showed that both miR-132 and miR-212 were significantly increased in the penumbral area $24 \mathrm{~h}$ after MCAO compared with the sham group (Supplemental Fig. 1). Visual stimulation induces histone modification (acetyl-lys9 H3) on the CRE loci upstream of the miR-132 and miR-212 coding sequences and activates mature and primary miR-132 expression [8]. To examine the functional role of CRTC1 in the regulation of miR-132/212 in cerebral ischemia, we generated CRTC1 KO mice using CRISPR/Cas9, and Western Blot showed that CRTC1 expression was completely absent in the mouse (Fig. 1A). No differences were found between CRTC1 KO and WT mice in 8week-old weight (Supplemental Fig. 2A), blood pressure (Supplemental Fig. 2B), and CBF under resting conditions (Supplemental Fig. 2C). qPCR showed no significant change in miR-132/212 expression in the nonischemic brain (Fig. 1B, C), while a significant reduction in miR-132/212 expression was detected in the CRTC1 KO penumbral area compared with WT $24 \mathrm{~h}$ after focal cerebral ischemia (Fig. 1D, E).

\section{CRTC1 deficiency aggravates ischemic brain injury}

To clarify the role of CRTC1 in ischemic stroke, we subjected both CRTC1 KO and WT mice to $60 \mathrm{~min}$ of MCAO and evaluated the infarct volume using TTC staining after $24 \mathrm{~h}$ (Fig. 2A). Strikingly, the CRTC1 KO mice showed an approximately twofold increase in ischemic stroke volume (Fig. 2B) compared with WT mice. Consistent with this finding, CRTC1 KO mice exhibited a twofold higher death rate compared with WT mice $24 \mathrm{~h}$ after MCAO (Supplemental Table 1). We also monitored cerebral blood flow (CBF) reperfusion in both CRTC1 KO and WT mice (Fig. $2 \mathrm{C}$ and Supplemental Fig. 2C) and found although no significant CBF changes were found until $10 \mathrm{~min}$ after the operation, CBF reperfusion was worsened in CRTC1 KO mice compared with WT mice $24 \mathrm{~h}$ after MCAO (Fig. 2D). To determine whether this difference in $\mathrm{CBF}$ reperfusion was caused by BBB breakdown, we examined $\mathrm{BBB}$ integrity using Evans Blue dye (Fig. 2E and Supplemental Fig. 3). Compared with the nonischemic hemisphere, there was an increase in Evans Blue staining in the ischemic hemisphere in both CRTC1 KO and WT mice, but the accumulation of Evans Blue was greater in the CRTC1 KO compared with the WT (Fig. 2F). These results suggest that CRTC1 deletion increases ischemic stroke volume and worsens BBB leakage induced by MCAO.

Next, we examined the effect of CRTC1 knockout on mouse behavioral outcomes after stroke. Both CRTC1 KO and WT mice exhibited significant functional deficits after MCAO (Fig. 2G, H). Compared with WT mice, CRTC1 KO mice showed a statistically worse neurological score (Fig. 2G), and significant worse forelimb use in both the foot fault test (Fig. 2H). We also performed rotarod test and cylinder test for behavior assessment, but failed to distinguish differences. This may because that mice showed extremely weakness for adherence to drum in the rotarod test, and barely spontaneous forelimb use for vertical movement in cylinder test. This is consistent with a previous report [24]. These results suggest that CRTC1 deletion aggravates ischemic stroke outcome, without altering cerebral anatomical structures.

\section{CRTC1 deficiency and miR-132 knockdown aggravate neuronal damage via RBFox-1}

The impact of CRTC1 deletion on stroke outcomes led us to hypothesize that CRTC1 affects NVU integrity by regulating 


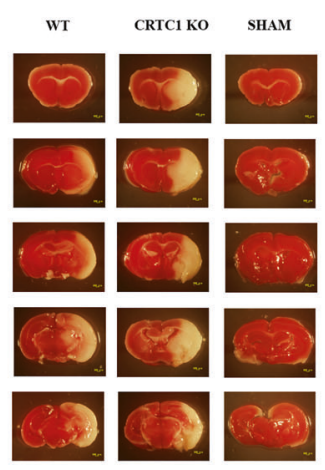

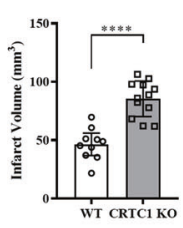

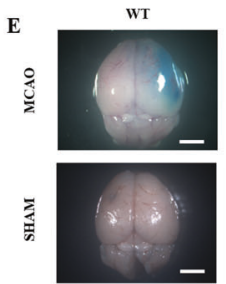

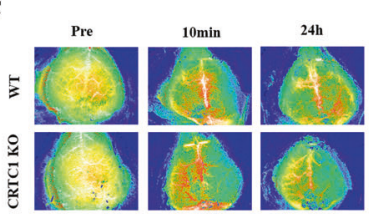

CRTC1 ко

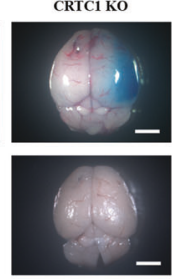

F

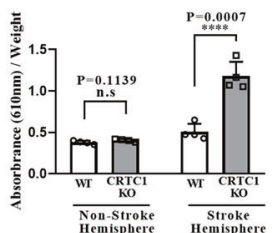

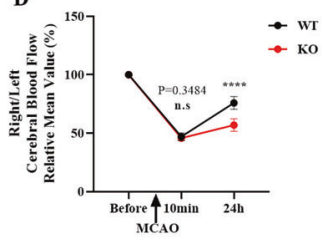

G

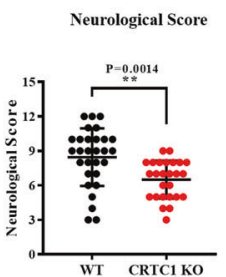

H

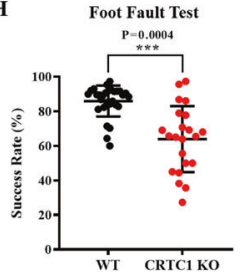

Fig. 2 CRTC1 deficiency exaggerates blood-brain barrier breakdown and mice forelimb recovery after ischemia. A Representative TTC staining images of CRTC1 KO and WT mice brain sections $24 \mathrm{~h}$ after MCAO. B Quantification of infarct volume of CRTC1 KO and WT mice. $n=$ 10 for each group. C Cerebral blood flow (CBF) pictures of CRTC1 KO and WT mice before and after MCAO. D Quantification of relative CBF change of right/left MCA responsible area. $n=10$ for each group. Representative images $(\mathbf{E})$ and quantification $(\mathbf{F})$ of Evans Blue extravasation in CRTC1 KO and WT mice brains after MCAO. $n=4$ for each group. Mice behavior deficits $24 \mathrm{~h}$ after MCAO were examined by Neurological Score (G) and Foot fault Test (H). $n=29$ for WT, $n=26$ for CRTC1 KO.

A
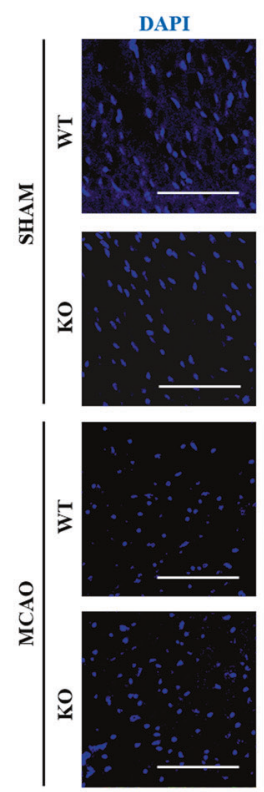

Neun
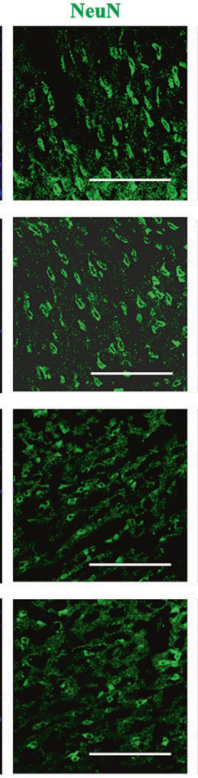

Caspase-3
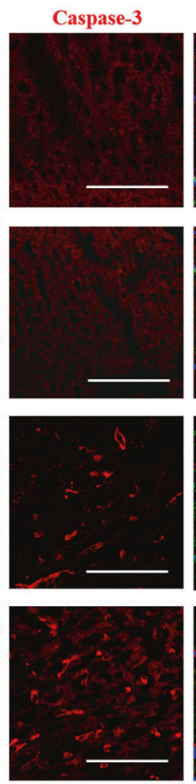
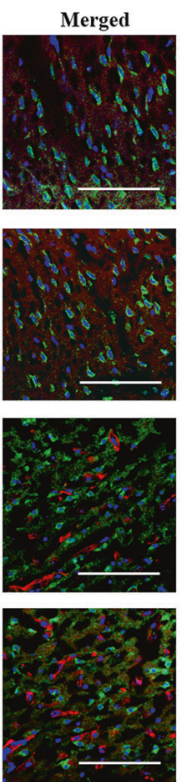

D

$\mathbf{E}$
B

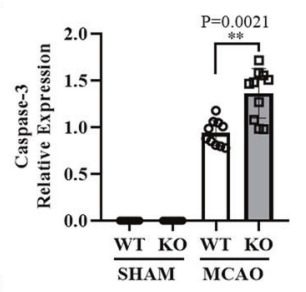

C

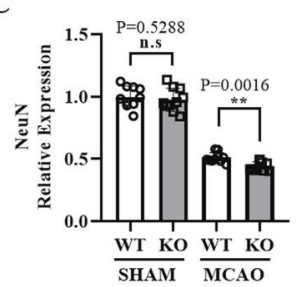

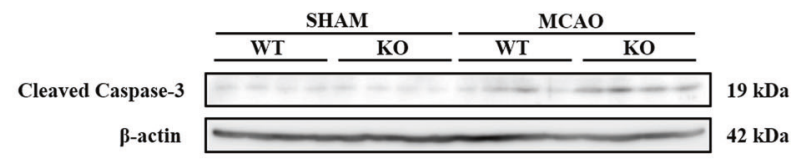

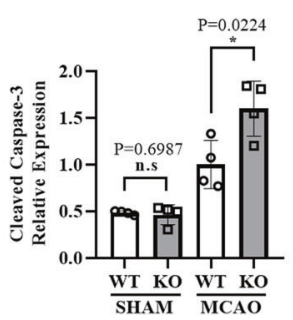

Fig. 3 CRTC1 deletion and miR-132 knockdown aggravate neuron apoptosis. Representative immunostaining images (A) and quantification of Caspase-3 (B) and NeuN (C) in CRTC1 and KO brain sections. (Scale Bar, $100 \mu$ m). Representative western Blot image (D) and quantification (E) of cleaved caspase-3 in CRTC1 KO and WT penumbra regions.

miR-132/212. RBFox-1, which is reported to be critical for neuronal activity, was predicted to be a miR-132/212 target by bioinformatics analysis. In order to investigate neuron apoptosis in vivo, we applied NeuN and Caspase-3 immunostaining to CRTC1 KO and WT mice brain sections (Fig. 3A). We found CRTC1 KO mice showed an enhanced Caspase-3 expression (Fig. 3B) and a relatively decreased NeuN expression (Fig. 3C) compared to WT mice in cortex of penumbra area. Western Blot (Fig. 3D) showed an elevated cleaved Caspase-3 (Fig. 3E) in CRTC1 KO mice after $\mathrm{MCAO}$, indicating a higher apoptosis in CRTC1 KO mice.

To further investigate the mechanism underlying damage induced by CRTC1 deletion in neurons, we subjected primary cortical neurons derived from CRTC1 KO and WT mice to Oxygenglucose deprivation (OGD). The CRTC1-deficient neurons displayed significantly exacerbated ischemic damage compared with wild type, as shown by MAP2 immunostaining (Fig. 4A) and LDH assay (Fig. 4B). We examined the expression of RBFox- 1 in both CRTC1 KO and WT mice under SHAM and ischemic conditions (Fig. 4C). Although no significant difference of RBFox-1 expression was observed between CRTC1 KO and WT mice under SHAM condition, MCAO induced RBFox-1 expression decrease in both CRTC1 KO and WT mice, while CRTC1 deficiency attenuated this decrease (Fig. 4D). To investigate whether RBFox-1 mRNA is regulated by miR-132 after ischemia, we generated a $\mathrm{CHO}$ line stably expressing the RBFox-1 3'-UTR (Fig. 4E) and transfected this cell line with miR-132 mimic, antagomir, and vehicle. We observed that miR-132 mimic significantly downregulated RBFox-1, while miR-132 antagomir transfection attenuated this decrease, especially after OGD/Reperfusion in CHO cells stably expressing the RBFox-1 3 '-UTR. This suggests that miRNA-132 regulates RBFox-1 
A

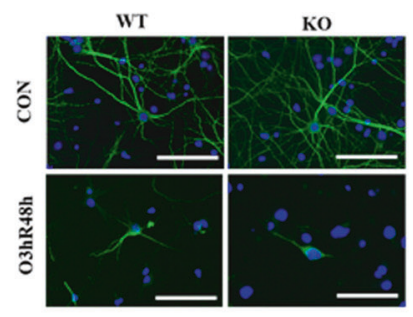

C

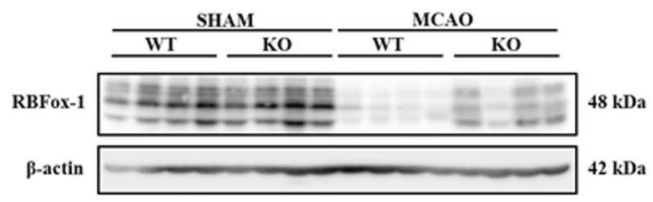

F

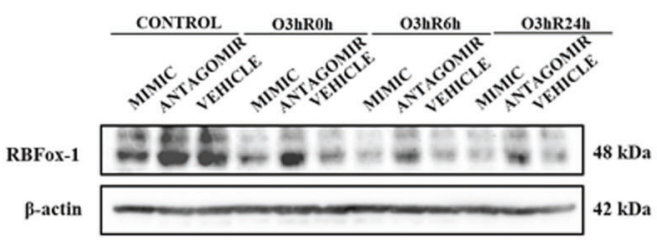

B

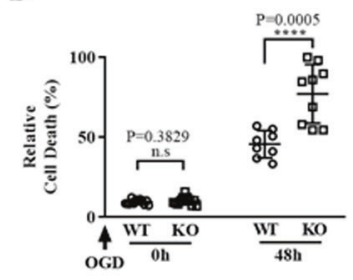

D

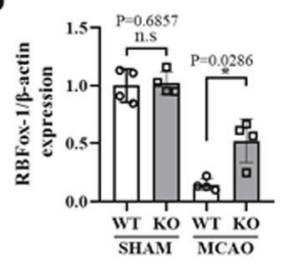

E

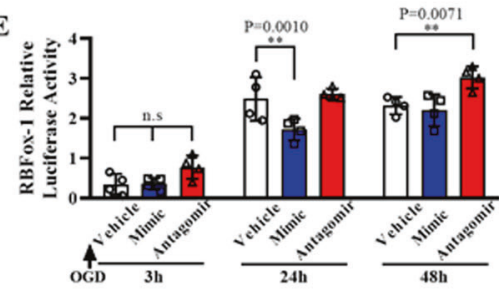

G

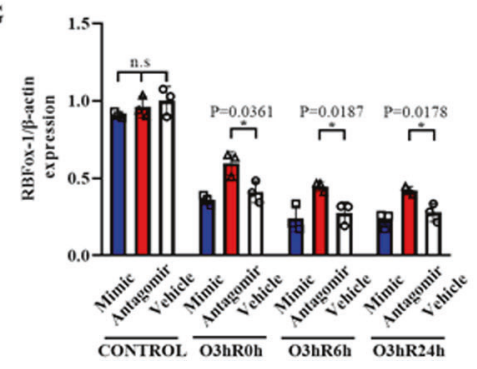

Fig. 4 CRTC1 deletion and miR-132 knockdown aggravate neuron damage. A Representative microtubule-associated protein 2 (MAP2) immunostaining images of CRTC1 KO and WT neurons, before and after OGD. (Scale Bar, $100 \mu \mathrm{m}$ ). B LDH assay of CRTC1 KO and WT neuron up to $48 \mathrm{~h}$ after ischemia/reperfusion. Representative Western Blot images (C) and quantification (D) of RBFox-1 in CRTC1 KO and WT mice penumbra region. E Relative luciferase activity of $\mathrm{CHO}$ cell line co-transfected with RBFox-1 and miR-132 vehicle/mimic/antagomir. Representative Western Blot images $(\mathbf{F})$ and quantification $(\mathbf{G})$ of RBFox-1 in neurons transfected with miR-132 mimic/antagomir/vehicle, before and after OGD.

mRNA under ischemic conditions. To date, there are no published reports of RBFox-1-mediated splicing changes after cerebral infarction. Here, we show, for the first time, changes in RBFox-1 after stroke, both in vivo and in vitro, and the role of CRTC1-miR$132 / 212$ in these changes. QPCR revealed no significant changes in RBFox-2 or RBFox-BS in mouse brains (Supplemental Fig. 4). Furthermore, to confirm that the RBFox- 1 expression change was caused by miR-132/212, we transfected neurons with miR-132 mimic, antagomir or vehicle, and then subjected these cultures to OGD, with or without subsequent reperfusion (Fig. 4F). No statistically significant differences in RBFox-1 expression were observed among these groups under control conditions. Antagomir significantly repressed the inhibition by miR-132 on RBFox-1 expression, while mimic slightly promoted the inhibition compared to the vehicle group after OGD/Reperfusion (Fig. 4G). Taken together, these results suggest that CRTC1 deletion and miR-132/ 212 knockdown both aggravate post-stroke neuronal damage and upregulate RBFox-1 in neurons.

\footnotetext{
CRTC1 deficiency suppresses vascular bed and destabilizes tight junction protein expression in the ischemic mouse brain To investigate whether CRTC1 deletion leads to endothelial cell dysfunction, we performed Lycopersicon esculentum lectin immunofluorescence staining to examine CRTC1 KO and WT brain vascular formation (Fig. 5A). No statistically significant change was observed in penumbra between the two groups under control conditions, and cerebral ischemia led to vasculature damage in the penumbra in both CRTC1 KO and WT mice. However, CRTC1 $\mathrm{KO}$ mice exhibited aggravated vascular bed damage compared with WT (Fig. 5B).
}

In the adult mammalian brain, interactions among several tight junction proteins, such as claudin- 5 and adhesive junction protein $\mathrm{E}-\mathrm{Cadherin}$, contribute to BBB integrity. To examine the mechanism underlying endothelial damage induced by CRTC1 deletion, we performed western blot for tight junction proteins in CRTC1 KO and WT penumbra. TJAP-1, which is demonstrated to be critical for BBB integrity, was also evaluated (Fig. 5C). In the acute phase after stroke, CRTC1 KO mice exhibited a statistically significant increase in TJAP-1 expression (Fig. 5D) and Claudin-1 expression (Fig. 5E) in comparison with WT mice. We also examined E-cadherin expression (Fig. 5F) in CRTC1 KO and WT brain, but no significant differences were observed. We next examined tight junction protein expression in mouse brain microvascular endothelial cells (BMECs) transfected with miR-132 mimic, antagomir, or vehicle, before and after OGD (Fig. 5G). An overall upregulation of Claudin1 was observed after OGD/reperfusion, while antagomir increased specially Claudin- 1 expression and mimic reduced slightly Claudin1 expression compared to the vehicle group (Fig. 5H). These results suggest that miR-132 regulates Claudin-1 mRNA under ischemic condition.

In CRTC1 KO-derived BMECs, the expression of Caludin-1 mRNA was significantly higher than that in the wild type, especially after OGD/Reperfusion. Next, we performed immunofluorescence staining for Claudin-1, Claudin-5, and ZO-1 in BMECs, under control and ischemic conditions (Fig. 6A, B). Ischemia dramatically increased Claudin-1 expression in BMECs, while KO BMECs showed an enhanced upregulation of Claudin-1 compared with WT (Fig. 6C). We also evaluated the Claudin-5 (Fig. 6D) and ZO-1 (Fig. 6E) expression in BMECs, but no statistically significant differences were found between KO and WT, before or after OGD. To further elucidate the regulatory role of miR-132/212 in endothelial cells, 

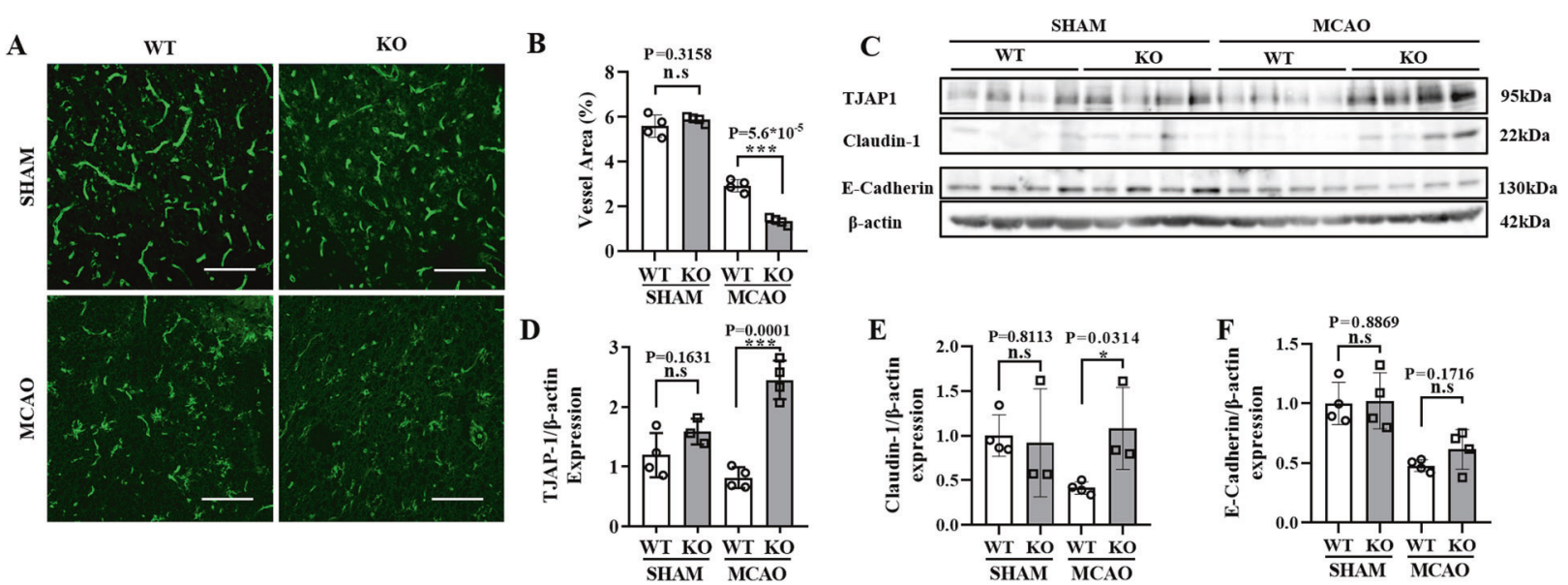

G

H

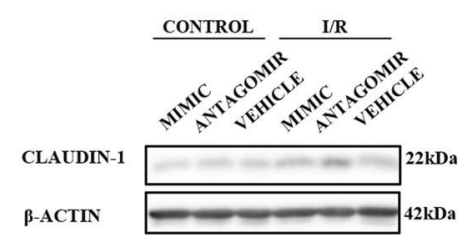

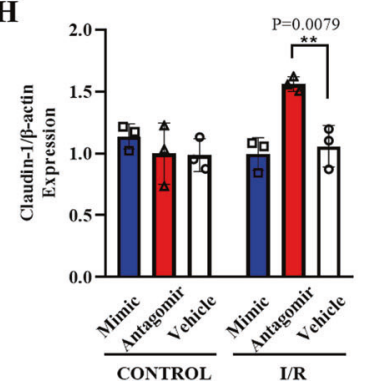

Fig. 5 CRTC1 deficiency suppresses vascular bed and destabilizes tight junction proteins in ischemic mice brains. Representative images (A) and quantification (B) of Lycopersicon esculentum Lectin immunofluorescence staining of mice penumbra (Scale Bar, $100 \mu \mathrm{m}$ ). Representative western blot images (C) and quantification of TJAP-1 (D), Claudin-1 (E), and E-Cadherin (F) expression after MCAO. Representative Western Blot images (G) and quantification (H) of Claudin-1 in mouse brain microvascular endothelial cells (BMECs) transfected with miR-132 mimic/antagomir/vehicle, before and after OGD.

we transfected Human umbilical vein endothelial cells (HUVECs) with miR-132 mimic, antagomir, or vehicle, before or after OGD (Supplemental Fig. 5). Claudin-1 was not detected in HUVECs before or after OGD, and miR-132 transfection did not statistically alter Claudin-5 or ZO-1 expression compared with the control group. To elucidate the functional role of CRTC1 in tight junction protein expression, Claudin-1 qPCR was performed in BMECs derived from both wild-type and CRTC1 KO mice after ischemia (Fig. 6F). qPCR showed Claudin-1 expression was obviously elevated $3 \mathrm{~h}$ after OGD in both CRTC1 KO and wild-type BMECs. Collectively, these data suggest that CRTC1 deletion and miR-132/ 212 inhibition both increase Claudin-1 and TJAP-1 expression in endothelial cells after ischemia.

\section{MiR-132/212 translationally enhances BBB integrity in ischemia}

To clarify the mechanism by which miR-132/212 regulates TJAP-1 and Claudin-1 expression, we conducted bioinformatics analysis and dual-luciferase assay. Bioinformatics analysis indicated one conserved miR-132/212 binding site within the $3^{\prime}$-UTR of mouse TJAP-1 mRNA (Fig. 7A) and four conserved miR-132/212-binding site within the $3^{\prime}$-UTR of mouse Claudin-1 mRNA (Fig. 7B), suggesting that miR-132/212 might translationally repress these tight junction proteins by direct binding to their $3^{\prime}$-UTR mRNAs. Therefore, we transfected pmiRGLO vector containing each $3^{\prime}$-UTR sequence into $\mathrm{CHO}$ cells to generate a stable cell line expressing $3^{\prime}$-UTR of these genes (wild-type or mutant type), then cotransfected these $\mathrm{CHO}$ cells with miR-132 mimic, miR-132 antagomir or vehicle. We found transfection of miR-132 mimic into $\mathrm{CHO}$ significantly decreased the luciferase activity of the wildtype 3'-UTR of mouse TJAP-1 and Claudin-1, while transfection of miR-132 antagomir into $\mathrm{CHO}$ significantly increased the luciferase activity of the wild-type $3^{\prime}$-UTR of mouse Claudin-1 (Fig. 7C, D).
Importantly, neither miR-132 mimic nor antagomir transfection showed any statistically significant effects on the luciferase activity in $\mathrm{CHO}$ that contains mutation of $468-470$ bp within mouse TJAP$13^{\prime}$-UTR and that contains mutation of 1844-1846 bp within mouse Claudin-1 3'-UTR. Collectively, these findings suggest miR132 specifically suppress TJAP- 1 and Claudin- 1 expression by directly binding to their $3^{\prime}$-UTR mRNA regions. To investigate the role of miR-132/212 in endothelial cell function in ischemia, we measured the transendothelial electrical resistance (TEER) values of HUVECs transfected with miR-132 mimic, antagomir or vehicle, followed by OGD and up to $48 \mathrm{~h}$ of reperfusion (Fig. 7E). MiR-132 mimic significantly attenuated the decline in TEER values after OGD in comparison with vehicle (Fig. 7F).

As described in previous studies, both CRTC1 and miR-132/212 are selectively expressed in neurons. Previous studies show that neurons secrete miR-132 to endothelial cells in zebrafish under ischemic stroke [25]. We observed that miR-132 levels in BMECs were not different between wild-type and CRTC1 KO, before and after OGD (Supplemental Fig. 6A). Western blotting suggested that CRTC1 is abundantly expressed in neurons compared with endothelial cells (Supplemental Fig. 6B). To elucidate the functional role of miR-132/212 in the BBB in ischemia, we modeled the BBB in vitro, using a co-culture system of vascular endothelial cells (HUVECs) and neurons derived from CRTC1 KO or WT mice (Fig. 7G). We assessed the endothelial cells' resistance to OGD by TEER, up to $48 \mathrm{~h}$ after reperfusion. HUVECs co-cultured with CRTC1deficient neurons exhibited a progressively exacerbated barrier breakdown after OGD, compared with HUVECs co-cultured with WT neurons (Fig. $7 \mathrm{H}$ ). To specify the direct relationship between neuron-derived miR-132 and TJPs, we seeded HUVECs in six-well transwell inserts, co-cultured with or without primary neurons. Cultures were subjected to $3 \mathrm{~h}$ OGD following $24 \mathrm{~h}$ reperfusion, then applied to Western Blot assay (Fig. 7l). After $24 \mathrm{~h}$ reperfusion 
A

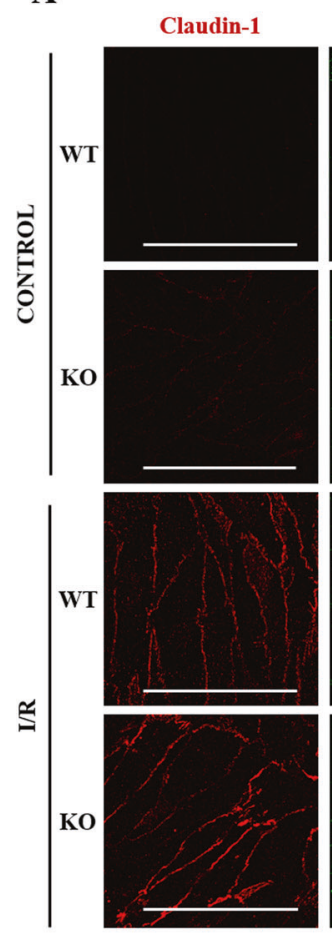

C

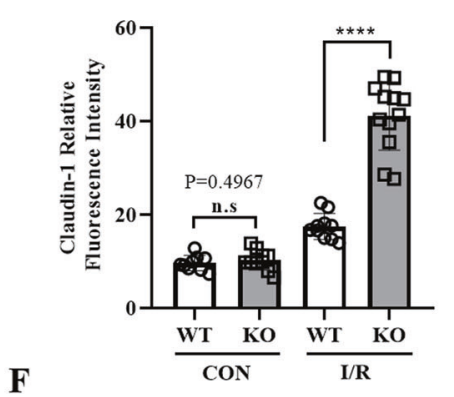

ZO-1
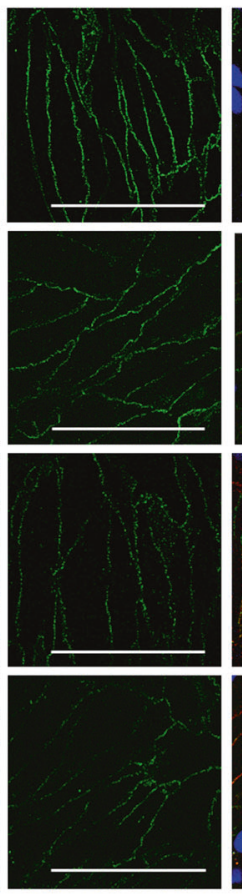

$\longrightarrow$$$
\text { I/R }
$$

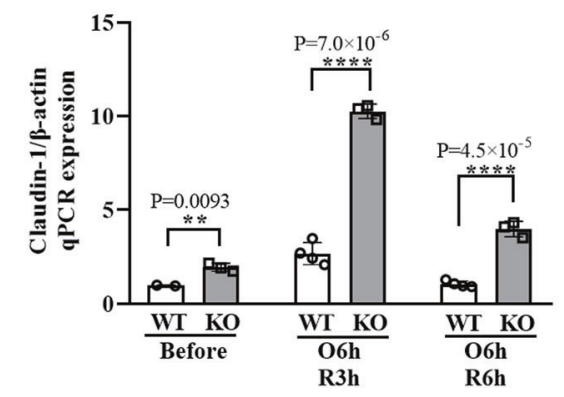

B
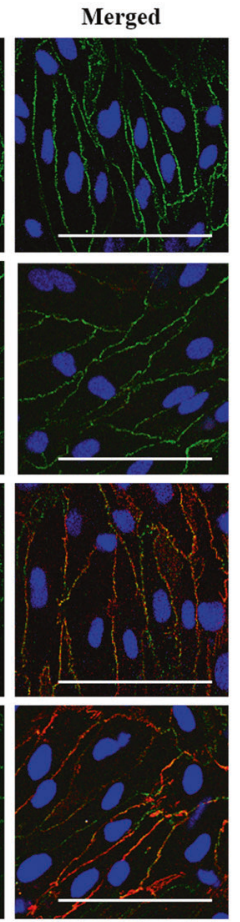

D

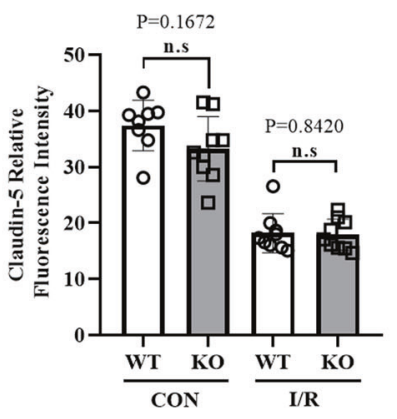

Claudin-5
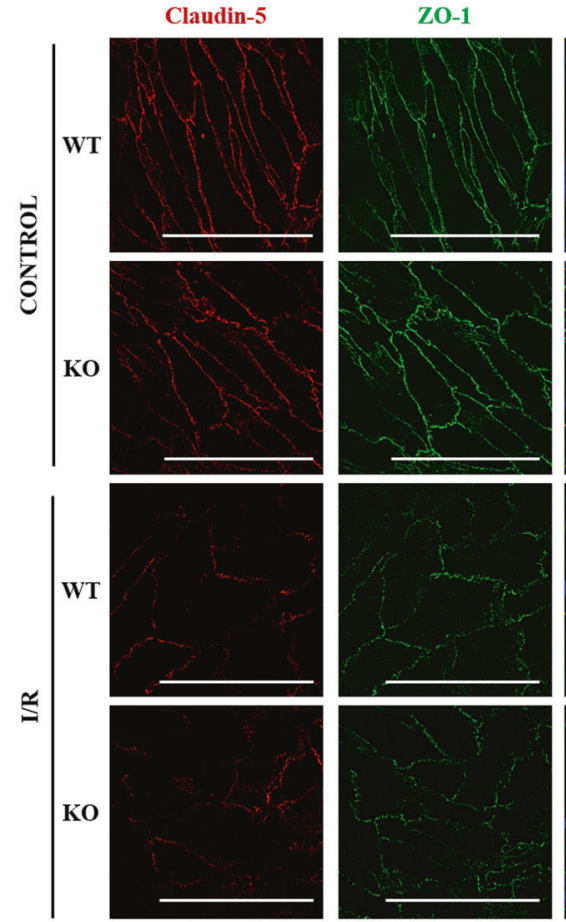

E

$\breve{s}$

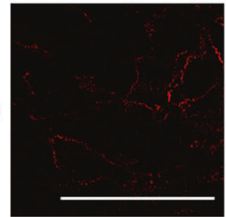

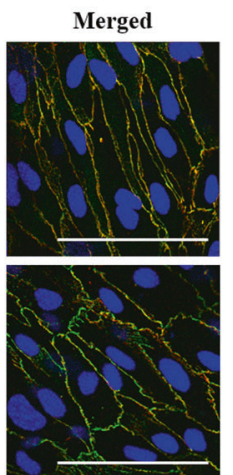
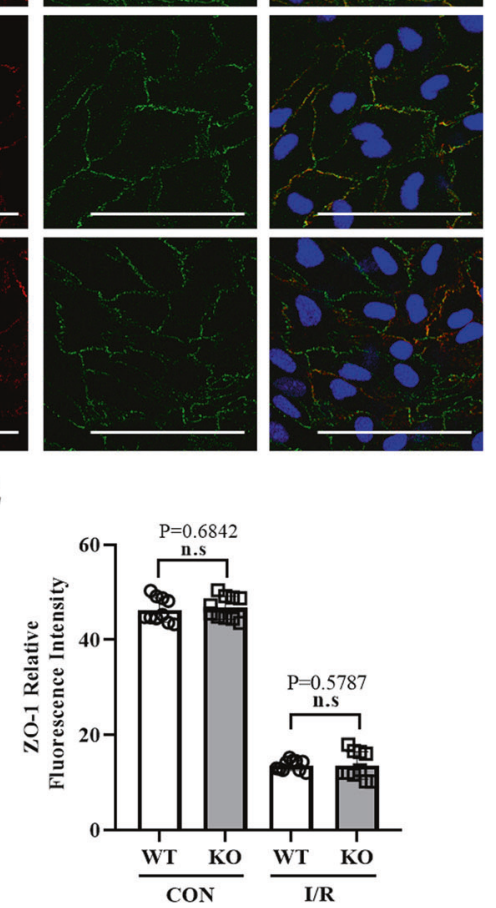

Fig. 6 CRTC1 deficiency enhances TJ proteins destabilization induced by ischemia/reperfusion in Mouse BMECs. Representative immunostaining images of Claudin-1/ZO-1 (A), Claudin-5/ZO-1 (B) and quantification of Claudin-1 (C), Claudin-5 (D), and ZO-1 (E) of BMECs derived from CRTC1 KO and WT, before and after ischemia/reperfusion (Scale Bar, $100 \mu \mathrm{m}$ ). F qPCR results of Claudin-1 in BMECs derived from CRTC1 KO and WT mice, before and after ischemia/reperfusion. $n=4$ for each group.

following $3 \mathrm{~h}$ OGD, HUVEC co-cultured with neurons showed a relatively lower expression of TJAP-1 (Fig. 7J) and Claudin-1 (Fig. 7K) than HUVECs cultured alone. To functionally test the paracrine secretion of miR-132 from neuron, we transfected miR-132/212 mimic/antagomir/vehicle into neurons which were co-cultured with HUVECs, then subjected the cultures to OGD following up to $48 \mathrm{~h}$ reperfusion (Fig. $7 \mathrm{~L}$ ). No significant differences were found before OGD among these groups. After subjected to OGD/ Reperfusion, HUVECs co-cultured with neurons that were transfected with miR-132 mimic gradually attenuated the TEER decline compared to the vehicle group, suggesting that miR-132 upregulated ECs resistance to OGD by an external pathway. HUVECs co-cultured with neurons that were transfected with antagomir showed hardly differences compared to the vehicle group, which may because OGD/Reperfusion inducing ECs damage was already occurred that could be hardly exaggerated furthermore (Fig. 7M). Taken together, these results indicate that CRTC1 affects BBB integrity by a paracrine pathway, suggesting that CRTC1-mediated release of miR-132/212 from neurons impacts the functional integrity of vascular endothelial cells (Fig. 8). 
A

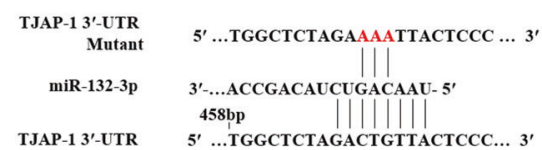

C

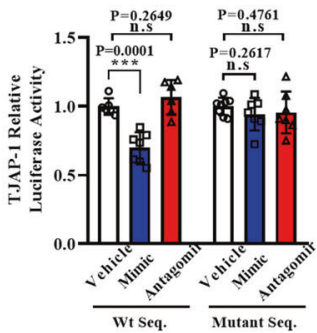

$\mathbf{E}$

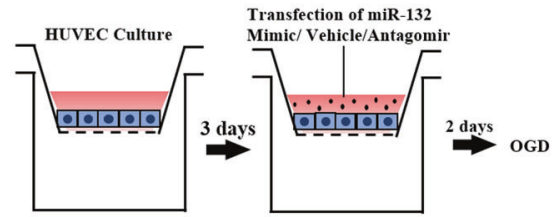

G
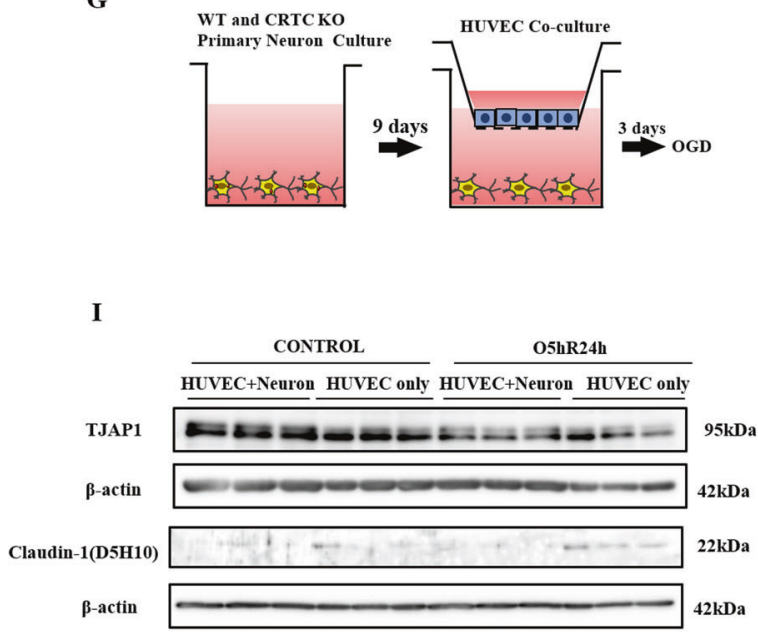

$\begin{array}{ll}\text { L WT Primary } & \begin{array}{l}\text { Transfection of miR-132 } \\ \text { Mimic/ Vehicle/Antagomir }\end{array}\end{array}$

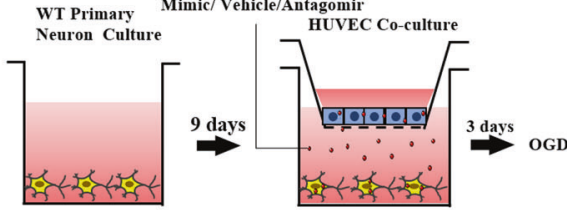

\section{DISCUSSION}

Maintaining BBB integrity is vitally important for therapies of cerebral ischemia. Here, we investigated the functional role of miR$132 / 212$ in neuronal/endothelial cell injury in cerebral ischemia. We found that CRTC1 affects miR-132/212 expression in mice, and we
B

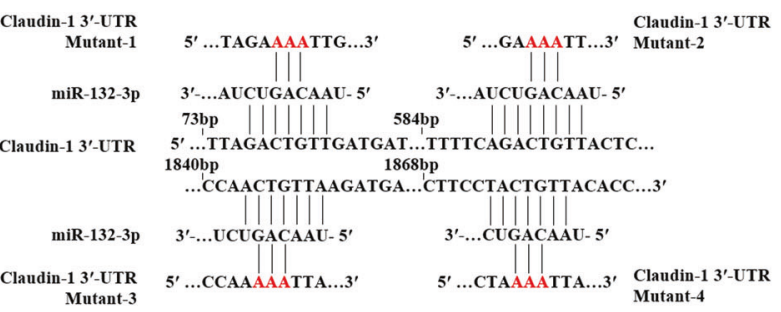

D

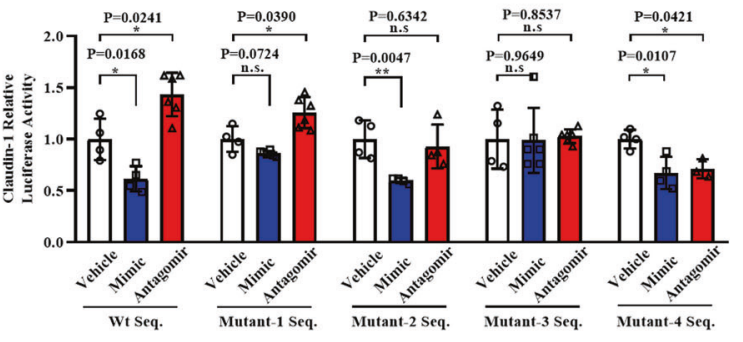

F

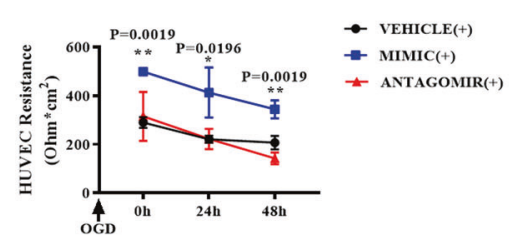

H
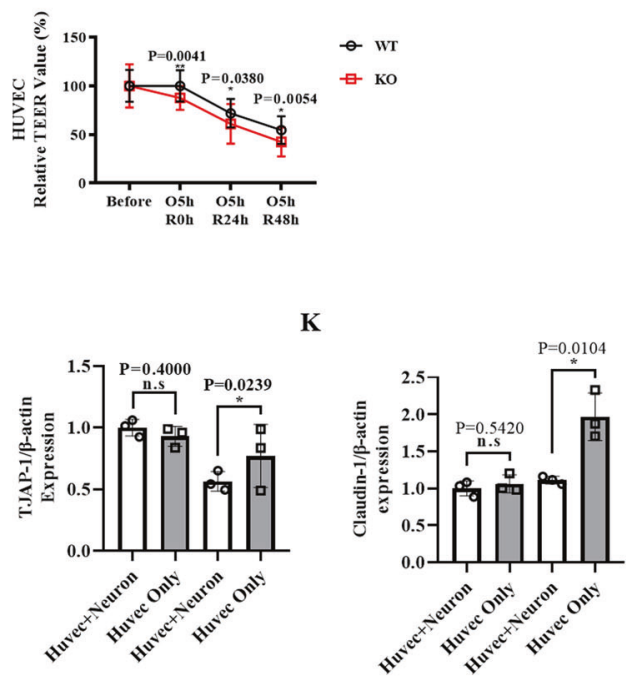

$\mathbf{M}$

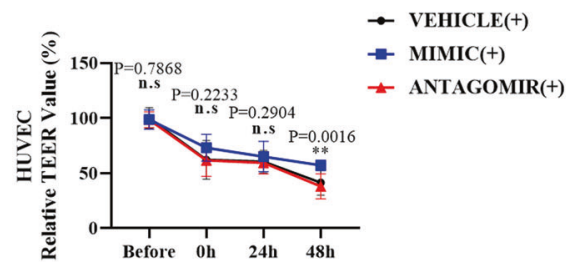

showed that miR-132 within the NVU regulates BBB homeostasis through an intercellular mechanism. In this study, we demonstrated that CRTC1 KO mice display a progressive downregulation of miR-132/212 in the early post-stroke phase, and exhibit aggravated neurological deficits and ischemic cerebral damage. 
Fig. 7 Neuron secretes miR-132/212 to endothelial cells to alleviate BBB breakdown after ischemia/reperfusion. The partial sequence of miR-132 and its one putative binding site in the $3^{\prime}$-UTR of the mouse TJAP-1 (A) and four putative binding sites in the $3^{\prime}-$ UTR of the mouse Claudin-1 (B), with each mutant 3'-UTR. Quantitative data showed that transfection of miR-132 mimic decreased luciferase activity of the reporter vector containing wild-type $3^{\prime}$-UTR sequence of mouse TJAP-1 (C) and Claudin-1 (D), while transfection of miR-132 mimic or antagomir had no effects on the luciferase activity of the reporter vector containing mutation of 468-470 bp within mouse TJAP-1 3'-UTR (C) and mutation of 1844-1846 bp within mouse Claudin-1 3'-UTR (D). (E) HUVECs were transfected with miR-132 mimic/vehicle/antagomir, then subjected to OGD. (F) TEER evaluation indicates that miR-132 mimic significantly attenuated the decline in TEER values after OGD, while miR132 antagomir aggravated HUVEC resistance to hypoxia. $n=3$ for each group. (G) A BBB model in vitro was constructed by a co-culture system of HUVECs and neurons derived from CRTC1 KO and WT mice. (H) HUVECs co-cultured with CRTC1 KO neurons exhibited progressively decreased TEER values after OGD compared with HUVECs co-cultured with WT neurons. Representative Western Blot images (I) and quantification of TJAP-1 (J) and Claudin-1 (K) of HUVECs co-cultured with or without neurons, before and after OGD. (L) HUVECs were cocultured with neurons that were transfected with miR-132 mimic/antagomir/vehicle, then subjected to OGD. (M) TEER evaluation indicates that miR-132 mimic by paracrine secretion significantly attenuated the decline in ECs TEER values after OGD. $n=10$ for each group.

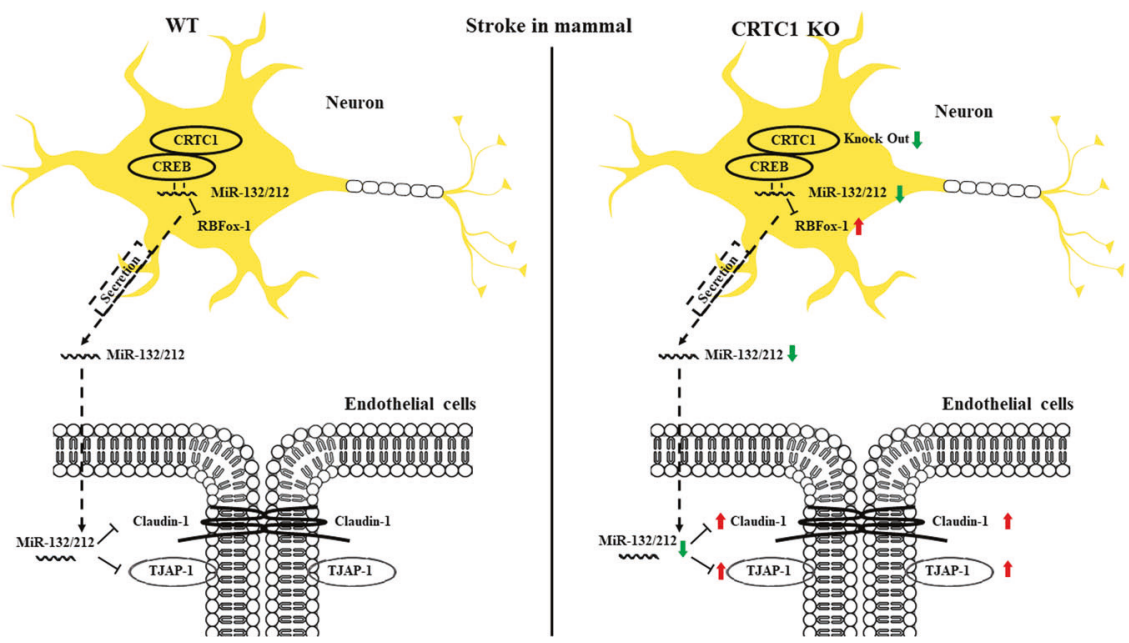

Fig. 8 Graphic abstract. CRTC1-mediated miR-132/212 attenuates neuron damages via suppressing RBFox-1, and alleviates blood-brain barrier dysfunction by regulating TJAP-1 and Claudin-1 expression in ischemic stroke.

Considering that our previous study identified CRTC1 regulated neuron survival via BDNF and PGC-1a [12], here we found that CRTC1-modulated miR-132/212 protects against neuronal injury by modulating RBFox-1. Furthermore, miR-132/212 released from neurons enters endothelial cells and acts on tight junction proteins, such as Claudin-1 and TJAP-1.

MiR-132/212 is a well-studied miRNA cluster in CNS disorders such as neurogenerative diseases [26] and intracerebral hemorrhage [27]. CREB-regulated transcription coactivator (CRTC) includes CRTC1, CRTC2, and CRTC3, among which CRTC2 has been demonstrated to dictate the vascular endothelial function [28], and CRTC1 is a specifically expressed protein in neuron. CRTC1 has been suggested to act as a critical role in several neurodegenerative diseases, such as Alzheimer's disease [29-31], and Huntington's disease [32, 33]. Nucleus-specific CRTC1 activation in the hippocampus enhances contextual fear memory [34]. In this study, we predicted that miR-132/212 was regulated by CRTC1. Consistent with this hypothesis, we found that CRTC1 deficiency reduced levels of miR-132/212 under ischemic conditions.

As several targets and signaling pathways may participate in the function of miR-132/212 in ischemia, we focused on the role of miR-132/212 on neuron-endothelial interaction and neurological deficits after cerebral ischemia. Alternative pre-mRNA splicing has a critical role in CNS gene expression. The RBFox proteins regulate the splicing of numerous transcripts in neurons, thereby affecting synaptic transmission and membrane stability [35]. Our findings show that miR-132/212 inhibits RBFox- 1 expression, but does not alter RBFox-2 or RBFox-3. To clarify whether the upregulation of RBFox-1 results from miR-132/212 inhibition, we transfected primary cortical neurons with miR-132 mimic or antagomir and subjected them to OGD. Inhibition of miR-132 was sufficient to significantly increase RBFox-1 expression.

BBB is a highly complex and dynamic structure that stringently regulates substance exchange by intricate interactions of tight junction proteins between endothelial cells [36]. Recently, Zuo et al. reported that miR-132 promotes BBB integrity by repressing MMP9 via directly binding to its $3^{\prime}$-UTR and increasing MMP9 downstream tight junction proteins which refer to VE-Cadherin and $\beta$-catenin, but not differing TJPs like Occludin. It should be noted that TJPs in our study refer to Claudin-1, Claudin-5, and TJAP-1, that are different from the TJPs from the former study. The tight junction complex is mainly constructed by the linear polymers, which are formed by interactions of Claudin proteins with each other $[37,38]$. ZO-1 is a scaffold protein essential for Claudins polymerization. Recently, increasing studies suggested that rather than the expression level change of one key TJ protein, but a destabilized organization of TJ complex is more responsible for BBB breakdown [39], such as Claudin-1 knockdown led to a dynamic TJ proteins destabilization and improve BBB permeability and functional recovery after stroke [23]. In addition to TJ, adherens junctions (AJs) complex is also a major junctional complex in BBB. AJs are composed primarily of cadherin proteins that mainly contribute to cell-cell adhesion and stability by linking to the actin cytoskeleton via $\beta$-catenin proteins $[40,41]$. $\beta$-Catenin signaling in endothelial $A J$ plays a crucial role in maintaining cellular polarity and is important for maintaining adult BBB integrity in stroke [42]. However, the precise role of $A J$ in BBB and the relationship between $\mathrm{AJ}$ and $\mathrm{TJ}$ in neurological disorders have not yet been elucidated, so further studies are needed. TJAP-1 is a peripheral membrane protein located in TJ complexes first reported in 2001 [43], but its function remains unclear. This study 
is consistent with the previous studies. Moreover, our work demonstrated that CRTC1 deficiency contributes to BBB destabilization by increasing Claudin-1 expression in BMEC after ischemia/reperfusion for the first time using immunofluorescence staining, and that inhibition of Claudin-1 by miR-132 improves BBB recovery after ischemia/reperfusion. We clarified the regulation of miR-132 on Claudin-1 by insert mutation into 1844-1846 bp within Claudin-1 3'-UTR. Although its role in BBB remains unclear, TJAP-1 is presumed to have a regulatory role in BBB integrity because of its localization toward the cytoplasmic face of TJs and within the Golgi stack [44]. In this study, TJAP-1 and Claudin-1 exhibited a significant increase in CRTC1 KO mice compared with WT mice, leading to BBB damage by Claudin-5/Claudin- 1 dysregulation in the CRTC1 KO brain after stroke. Furthermore, our observation of HUVECs transfected with miR-132 mimic/ antagomir revealed that miR-132 directly regulates Claudin-1 and TJAP-1 in endothelial cells. Using TEER, we found that transfection of miR-132 antagomir significantly reduced the resistance of HUVECs to hypoxia, indicating that miR-132 directly regulates endothelial cell interactions.

Next, as both CRTC1 and miR-132/212 are neuron-specifically expressed, we investigated whether intercellular interaction between neurons and endothelial cells maintain neurovascular homeostasis. BBB functions depend mostly on the perivascular microenvironment, especially on neuronal cells and BBB-forming endothelial cells. Under ischemic conditions, various factors are released from neurons to endothelial cells, in an attempt to attenuate BBB dysfunction. We investigated the role of miR-132/ 212 in neuron-endothelial cell interactions using a co-culture system. Consistent with the evidence that neurons secrete exosomes containing miR-132/212 to endothelial cells in zebrafish [25], we found that HUVECs cultured with CRTC1 KO neurons, which lack miR-132/212, showed reduced resistance to hypoxia. This suggests that CRTC1 in neurons regulates TJ protein interactions in endothelial cells via a paracrine signaling mechanism to maintain BBB integrity in Neurovascular unit after stroke.

The CRTC family was initially identified as co-activators of CREB via the dZIP domain, independent of the CBP/p300 pathway $[45,46]$. Unlike CRTC2 and CRTC3, CRTC1 is extremely highly expressed in the brain, and its expression level far exceeds that of CREB [47-49]. This suggests that CRTC1 has important and diverse functions in the brain [50].

Taken together, our findings demonstrate that CRTC1 deficiency aggravates neurological deficits in mice in the early phase after stroke. This worsening is mediated by RBFox-1 via disruption of CRTC1-miR-132 signaling in neurons. The miR-132/212 dysregulation impairs BBB function and is associated with perturbations in TJAP-1, Claudin-1, and Claudin-5. Further studies are needed to clarify the paracrine communication mechanisms by which miRNAs and non-coding RNAs affect CNS pathology after stroke. Investigation of the intercellular signaling mechanisms mediated by miRNAs may lead to novel approaches for the treatment of stroke.

\section{MATERIALS AND METHODS \\ Animal}

CRTC1 knockout was conducted with CRISPR/Cas9 on C57BL/6 background at Osaka University Genome Editing Research and Development (R\&D) Center [51]. Details were described in the Supplementary materials. Animals were raised under standard conditions of light (lights on: 8:00 a.m. to $8: 00$ p.m.) and temperature $\left(23^{\circ} \mathrm{C}, 40 \%\right.$ humidity). Systolic blood pressure was measured on 8 weeks old by a blood-pressure monitor (SOFTRON, Tokyo, Japan).

\section{Transient focal cerebral ischemic model}

Transient focal cerebral ischemia was conducted in 8-12 weeks old male mice by MCAO (MCAO) as described in our previous study [52]. Mice were randomly divided into SHAM and MCAO groups. General anesthesia was performed using isoflurane with an open mask, and cortical CBF was monitored by a laser-Doppler flowmetry from before to $10 \mathrm{~min}$ after operation. The right middle cerebral artery was occluded for $60 \mathrm{~min}$ with a suture followed by $24 \mathrm{~h}$ of reperfusion. Only mice with less than $30 \%$ of baseline control microperfusion during the first minute of occlusion were used in subsequent experiments. After the operation, mice recovered in their individual cages normally.

\section{Measurement of cerebral blood flow (CBF)}

The measurement of CBF was conducted as previously described [53]. Surface CBF was recorded by a laser speckle blood flow imaging system (Omegazone OZ-1). After general anesthesia, the skull was exposed by a midline scalp incision. The surface of the skull was wiped clean with salinesoaked gauze before recording. Color-coded CBF images were obtained in high-resolution mode. The mean CBF value was measured in identically sized regions of interest (900 pixels) located $3 \mathrm{~mm}$ posterior and $2.5 \mathrm{~mm}$ lateral from the bregma [53].

\section{2,3,5-triphenyltetrazolium hydrochloride (TTC) staining}

After $24 \mathrm{~h}$ of reperfusion, the brains were collected and sectioned at 2-mm intervals, and then stained with $2 \%$ TTC at room temperature for $1 \mathrm{~h}$. The brain sections were fixed in 4\% PFA for $30 \mathrm{~min}$, and photographed with a digital microscope (Olympus SZX12). The infarct volume was calculated as: whole contralateral hemisphere volume - nonischemic ipsilateral hemisphere volume.

\section{Evans Blue extravasation}

After $24 \mathrm{~h}$ of MCAO, $100 \mu \mathrm{l}$ of $4 \%$ Evans Blue was injected as a tracer into the circulation via the caudal vein. After $10 \mathrm{~h}$ of reperfusion, the brain was collected, and photographed before and after sectioned with a digital microscope (Olympus SZX12). The hemispheres were collected and homogenized respectively in $500 \mu \mathrm{l}$ formamide (Nacalai Tesque, Kyoto, Japan) at $60^{\circ} \mathrm{C}$ overnight. Supernatants were collected, and $100 \mu$ liquots were used for absorbance detection at $610 \mathrm{~nm}$. Formamide incubated at $60^{\circ} \mathrm{C}$ overnight was used as the blank, and the OD of each mouse was calculated as: average absorbance/hemisphere weight.

\section{Quantitative real-time PCR analysis}

For the miR-132/212 assay, total RNA, including small RNA, was extracted from neurons or tissues using the mirVana miRNA Isolation Kit (Thermo Fisher Scientific, Waltham, MA, USA). cDNA was prepared by reverse transcription (RT) from $1 \mu \mathrm{g}$ of total RNA using Taqman MicroRNA Reverse Transcription kit (Thermo Fisher Scientific). The RT products were used in real-time PCR analysis with the Taqman MicroRNA assay for miR-132 (Mm04238115_s1) and miR-212 (Mm04238228_s1), using the Taqman Fast Advanced Master Mix (Thermo Fisher Scientific). U6 RNA was used as normalization.

For relative mRNA transcript quantification, cDNA was prepared from $1 \mu \mathrm{g}$ total RNA using the SuperScript VILO cDNA Synthesis Kit (Invitrogen, Waltham, MA, USA). Power SYBR Green PCR Master Mix (Thermo Fisher Scientific) was used for real-time PCR. $\beta$-actin and 36B4 served as endogenous control. Primer sequences were described in Supplemental Table 2. Relative expression was calculated using the comparative CT method with the 7900HT Real-Time PCR System (Applied Biosystems, Waltham, MA, USA).

\section{Behavioral test}

The criteria used for neurological score were described in a previous study [54]. Spontaneous activity, symmetry of movements, symmetry of forelimbs, climbing wall of wire cage, reaction to touch on either side of trunk, and response to vibrissae touch were scored as 0-3 points, respectively. The lower score indicates worse neurological deficits. For the foot fault test, mice were placed on an elevated gridded platform above the surface and allowed to walk for $5 \mathrm{~min}$. A foot fault was noted when the left forefoot misstepped and fell through the space between the grids. The percentage of left foot faults was measured for statistical analysis during the whole observation. For the cylinder test, mice were placed inside a transparent cylinder with a tri-fold mirror placed behind to ensure both forelimbs could be seen. Forelimb use for vertical exploration was evaluated by counting left forelimb contacts on the cylinder. The success rate was obtained as: right forelimb failed contact/right forelimb 
contact + left forelimb contact + both contacts. For the rotarod test, the time at which mice fell off the drum was recorded as latency to fall. Mice alive but showed no spontaneous activity were excluded for the behavior test.

\section{Cell cultures and transfection}

Primary cultures of cortical neurons were prepared as described previously [55]. Briefly, neuronal cultures were prepared from the cortex of embryonic day 16 (E16) mouse embryos. Cortical tissue was incubated in dissection medium (Dulbecco's modified Eagle's medium containing $100 \mathrm{U}$ of papain and $0.5 \mathrm{mg} / \mathrm{ml}$ DNase type II) for $30 \mathrm{~min}$ at $37^{\circ} \mathrm{C}$. After centrifugation, cells were plated onto 24-transwell plates, 12-transwell plates, 6-transwell plates, 4-well culture slides, $60-\mathrm{mm}$ dishes, or 4-chamber glass slides (Corning, NY, USA) coated with polyethylenimine. Cells were cultured to a final concentration of $7.0 \times 10^{5}$ cells $/ \mathrm{ml}$ in high-glucose DMEM (Wako, Osaka, Japan) containing 10\% fetal calf serum (Invitrogen) and $100 \mathrm{IU} / \mathrm{ml}$ penicillin. After $24 \mathrm{~h}$, the medium was changed to Neurobasal medium (Invitrogen) supplemented with B-27 (Invitrogen). The cells were cultured at $37^{\circ} \mathrm{C}$ in a humidified atmosphere of $95 \%$ air and $5 \% \mathrm{CO}_{2}$ and used after 10-11 days in vitro when most cells displayed a neuronal phenotype. HUVECs were purchased from PromoCell (Heidelberg, Germany), and were cultured following the manufacturer's instructions.

BMEC isolation and purification were performed as described previously. Briefly, brain tissues were isolated from 3-week-old CRTC1 KO and WT mice and dissociated with $1 \mathrm{mg} / \mathrm{ml}$ collagenase type2 (Worthington, Columbus, $\mathrm{OH}$, USA). Then $20 \%$ BSA (Wako) in DMEM/F-12 (Wako) was used to remove myelin. The cell suspensions were incubated with $1 \mathrm{mg} / \mathrm{ml}$ collagenase/ dispase (Roche, Basel, Switzerland), and then gently injected onto Percoll (GE Healthcare, Chicago, IL, USA) for centrifugation at $1000 \times g$ for $10 \mathrm{~min}$. The endothelial cell layer was collected and cultured in DMEM/F-12 medium.

MiR-132 mimic, vehicle, and antagomir were purchased from Bioneer Cooperation (Daejeon, Korea). All procedures were conducted strictly as the manufacturer's instructions.

\section{Oxygen-glucose deprivation (OGD)}

OGD was conducted by placing cultures in an anaerobic atmosphere as previously described. Briefly, cultures were washed with phosphatebuffered saline (PBS) and incubated in glucose-free Earle's balanced salt solution (EBSS) (Biological Industries, Israel) in an anaerobic environment of $95 \% \mathrm{~N}_{2} / 5 \% \mathrm{CO}_{2}$, maintaining an $\mathrm{O}_{2}$ pressure of $10-15$ Torr, at $37^{\circ} \mathrm{C}$ for 2 or $3 \mathrm{~h}$. After incubation, OGD was terminated by replacing with complete medium and returning the cultures to a normoxic chamber.

\section{Cell viability assay}

To evaluate neuronal death, the Cytotoxicity Detection Kit (Roche) was used with the lactate dehydrogenase (LDH) assay at 0,24 , or $48 \mathrm{~h}$ after termination of OGD. For each culture, $200 \mu$ l of supernatant was transferred to a 96-well plate. After a brief centrifugation at $4{ }^{\circ} \mathrm{C}, 400 \times g$ for $10 \mathrm{~min}, 50 \mu \mathrm{l}$ of supernatant was transferred from each well to a new 96-well plate for the next step. The cytotoxicity detection buffer was mixed according to the manufacturer's instructions, and $50 \mu \mathrm{l}$ of mixture was added to the corresponding well using a multichannel pipette. The plate was wrapped with aluminum foil and incubated at room temperature for $30 \mathrm{~min}$. The $\mathrm{OD}$ was measured with an absorbance microplate reader (CORONA SH-9000Lab) at 490 and $650 \mathrm{~nm}$. Neuronal cytotoxicity was calculated as: $\mathrm{LDH}$ release $\left(\mathrm{OD}_{490}\right) / \mathrm{LDH}$ release $\left(\mathrm{OD}_{650}\right)$. One hundred percent cell death rate was revised to $2 \%$ TritonX-100 (Wako) pre-added in neuronal cultures before supernatant collection. Background control was recorded as absorbance value of $100 \mu \mathrm{l}$ cytotoxicity detection buffer mixture. Neuron death rate \% was calculated as (Example value - Background control)/(Maximum LDH releaseBackground control) [12].

\section{Immunofluorescence staining}

For immunofluorescence staining in vitro, neurons and HUVECs were cultured in four-well slides. Cultures were washed and fixed with 4\% PFA at room temperature for $30 \mathrm{~min}$. Slides were washed three times with PBS and then incubated with $0.3 \%$ TritonX-100 at room temperature for $30 \mathrm{~min}$. After 30 min of blocking with $10 \%$ donkey serum in TBS, slides were incubated with the following primary antibodies: MAP2 (1:500; Invitrogen), Claudin-5 (1:500; Abcam), Claudin-1 (1:500; Invitrogen) and ZO-1 (1:500; Invitrogen). Alexa Fluor 488-labeled donkey anti-mouse IgG, 647-labeled donkey anti-rabbit IgG, and 488-labeled donkey anti-rat IgG (Invitrogen) were used as secondary antibody.

For immunofluorescence staining in vivo, freshly isolated brains were covered in sufficient OCT embedding compound on dry ice, and cut into 16- $\mu \mathrm{m}$-thick sections. Sections were attached to slides and fixed with $1 \%$ PFA at room temperature for $10 \mathrm{~min}$, and incubated with $0.1 \%$ TritonX-100 at room temperature for $10 \mathrm{~min}$. After $30 \mathrm{~min}$ of blocking with $10 \%$ donkey serum in TBS, slides were incubated with 488 conjugated Lycopersicon esculentum tomato lectin (1:1000; Vector Laboratories, Burlingame, CA, USA) overnight at $4{ }^{\circ} \mathrm{C}$ in the dark. For immunofluorescence staining of NeuN and Caspase-3, mice were sacrificed after 2\% PFA perfusion, and brains were collected and cut into $16-\mu \mathrm{m}$-thick sections. Sections were incubated with $0.1 \%$ TritonX-100 at room temperature for $30 \mathrm{~min}$. After 30 min of blocking with $10 \%$ donkey serum in TBS, slides were incubated with NeuN (1:250; Novus Biologicals, Centennial, CO, USA) and Caspase-3 (1:250; Cell Signaling) overnight at $4{ }^{\circ} \mathrm{C}$, then subjected to $3 \mathrm{~h}$ incubation with secondary antibody (1:500; Invitrogen) including Alexa 488-labeled donkey anti-Mouse IgG and Alexa 594-labeled donkey anti-Rabbit IgG. Both cells and brain sections were counterstained with DAPI (Vector Laboratories), and a confocal laser-scanning microscope (Zeiss LSM-710) was used for visualization to avoid interference between channels and saturation.

\section{Western blotting}

Brains were collected in liquid nitrogen and lysed in TNE buffer on ice. Protein content was quantified with the BCA Protein Assay Kit (Thermo Fisher Scientific). Then, $10-\mu \mathrm{g}$ protein samples were electrophoresed and transferred to PVDF membranes (Merck Millipore, Burlington, MA, USA). After $1 \mathrm{~h}$ of blocking with $5 \%$ non-fat dry milk, membranes were incubated with primary antibody overnight at $4{ }^{\circ} \mathrm{C}$. After $1 \mathrm{~h}$ of secondary antibody incubation at room temperature, visualization was conducted with a luminoimage analyzer (ImageQuant LAS4000, GE Healthcare). For RBFox-1 normalization, membranes were subjected to WB Stripping solution (nacalai, Kyoto, Japan) after chemiluminescent exposure, then incubated with primary anti- $\beta$-actin antibody overnight at $4{ }^{\circ} \mathrm{C}$. The gray value of each lane was measured with Image-J software, and normalized to $\beta$-actin to obtain protein expression. The following antibodies were used: CRTC1 (1:1000; Cell Signaling Technology, Danvers, MA, USA); TJAP-1 (1:1000; Novus Biologicals); RBFox-1 (1:1000; Merck Millipore); E-Cadherin (1:1000; Cell Signaling Technology); Claudin-1 (1:1000; Invitrogen); cleaved Caspase-3 (1:1000; Cell Signaling Technology); $\beta$-actin (1:5000; Proteintech, Rosemont, IL, USA).

\section{Dual-luciferase reporter assay}

The miRNA databases miRBase (http://microrna.sanger.ac.uk) and TargetScan (http://www.targetscan.org) were used to identify potential miR-212/ 132 targets on BBB. the wild-type sequences of RBFox-1 $3^{\prime}-$ UTR, Claudin-1 $3^{\prime}$-UTR, and TJAP-1 $3^{\prime}$-UTR were constructed as Supplemental Table 3. Luciferase reporter genes were generated by inserting each $3^{\prime}$-UTR fragment downstream of the firefly luciferase gene in pmiRGLO, including TK-pRL (renilla) as an internal control. These pmiRGLO variants with mutations inserted into the miR-132/212 target sequences present in the $3^{\prime}$-UTR of TJAP-1 and Claudin-1 were obtained using a PrimeSTAR Mutagenesis Kit (Takara Bio, Shiga, Japan). Since the 3'-UTR of Claudin-1 has four assumed target sequences of miR-132, four mutants were made for each target sequence. The pmiRGLO vector containing each $3^{\prime}$-UTR fragment was transfected into $\mathrm{CHO}$ cells and a stable cell line expressing $3^{\prime}$-UTR of these genes was prepared by selection with G418. These stable $\mathrm{CHO}$ cell lines were co-transfected with miR-132 mimic, miR-132 antagomir, or vehicle (each $100 \mathrm{nM}$ ) using a NEPA21 electroporator (NEPA GENE). At $96 \mathrm{~h}$ post-transfection, the cells were subjected to a dualluciferase assay (Promega, Madison, WI, USA) as previously described.

\section{Transendothelial electrical resistance (TEER)}

A Millicell-ERS Volt-Ohm Meter was used, and all procedures were carried out as per the manufacturer's instructions. To investigate the role of CRTC1 in the interaction between neurons and endothelium, co-culture of neurons and HUVECs was performed. Neuronal cultures were plated onto 24-well plates as described above. After 9 days, HUVECs were seeded onto inserts (SARSTEDT, Nümbrecht, Germany) placed on top of the well containing neurons, at a density of $10^{4}$ cells/well. After 3 days, the cocultures were objected to OGD for $2.5 \mathrm{~h}$. TEER was performed at 0,24 , and $48 \mathrm{~h}$ after OGD. To investigate the direct effect of miR-132 on endothelium, 
HUVECs were seeded onto inserts at a density of $10^{4}$ cells/well. Transfections of miR-132 mimic, vehicle or antagomir were performed as described above 3 days after seeding. HUVEC cultures were objected to OGD for $3 \mathrm{~h}$, and TEER was performed at 0,24 , and $48 \mathrm{~h}$ after OGD.

\section{Statistical analysis}

Comparisons between two separate groups were performed using unpaired non-parametric $t$-test. Multiple groups comparisons were performed using one-way ANOVA with Bonferroni's post hoc test. All data are presented as means \pm standard deviation (SD).

\section{DATA AVAILABILITY}

The datasets generated and analyzed during the current study are available from the corresponding author on reasonable request.

\section{REFERENCES}

1. Sacco RL, Kasner SE, Broderick JP, Caplan LR, Connors JJ, Culebras A, et al. An updated definition of stroke for the 21st century: a statement for healthcare professionals from the American Heart Association/American Stroke Association. Stroke. 2013;44:2064-89.

2. Virani SS, Alonso A, Benjamin EJ, Bittencourt MS, Callaway CW, Carson AP, et al. Heart disease and stroke statistics-2020 update: a report from the American Heart Association. Circulation. 2020;141:e139-e596.

3. Marko M, Posekany A, Szabo S, Scharer S, Kiechl S, Knoflach M, et al. Trends of r-tPA (Recombinant Tissue-Type Plasminogen Activator) treatment and treatment-influencing factors in acute ischemic stroke. Stroke. 2020;51:1240-7.

4. Gebert LFR, MacRae IJ. Regulation of microRNA function in animals. Nat Rev Mol Cell Biol. 2019;20:21-37.

5. Li G, Morris-Blanco KC, Lopez MS, Yang T, Zhao H, Vemuganti R, et al. Impact of microRNAs on ischemic stroke: from pre- to post-disease. Prog Neurobiol. 2018;163-164:59-78.

6. Qian Y, Song J, Ouyang Y, Han Q, Chen W, Zhao X, et al. Advances in roles of miR132 in the nervous system. Front Pharmacol. 2017;8:770.

7. Zuo X, Lu J, Manaenko A, Qi X, Tang J, Mei Q, et al. MicroRNA-132 attenuates cerebral injury by protecting blood-brain-barrier in MCAO mice. Exp Neurol. 2019;316:12-9.

8. Tognini $P$, Putignano $E$, Coatti A, Pizzorusso T. Experience-dependent expression of miR-132 regulates ocular dominance plasticity. Nat Neurosci. 2011;14:1237-9.

9. Nudelman AS, DiRocco DP, Lambert TJ, Garelick MG, Le J, Nathanson NM, et al. Neuronal activity rapidly induces transcription of the CREB-regulated microRNA132, in vivo. Hippocampus 2010;20:492-8.

10. Balu DT, Li Y, Puhl MD, Benneyworth MA, Basu AC, Takagi S, et al. Multiple risk pathways for schizophrenia converge in serine racemase knockout mice, a mouse model of NMDA receptor hypofunction. Proc Natl Acad Sci USA. 2013;110:E2400-9.

11. Vo N, Klein ME, Varlamova O, Keller DM, Yamamoto T, Goodman RH, et al. A CAMP-response element binding protein-induced microRNA regulates neuronal morphogenesis. Proc Natl Acad Sci USA. 2005;102:16426-31.

12. Sasaki T, Takemori H, Yagita $Y$, Terasaki $Y$, Uebi T, Horike $N$, et al. SIK2 is a key regulator for neuronal survival after ischemia via TORC1-CREB. Neuron. 2011;69:106-19.

13. Won SY, Park MH, You ST, Choi SW, Kim HK, McLean C, et al. Nigral dopaminergic PAK4 prevents neurodegeneration in rat models of Parkinson's disease. Sci Transl Med. 2016;8:367ra170.

14. Wu Z, Huang $X$, Feng $Y$, Handschin $C$, Feng $Y$, Gullicksen PS, et al. Transducer of regulated CREB-binding proteins (TORCs) induce PGC-1alpha transcription and mitochondrial biogenesis in muscle cells. Proc Natl Acad Sci USA. 2006;103:14379-84.

15. Jiang $B$, Wang $H$, Wang JL, Wang $Y$ J, Zhu $Q$, Wang $C N$, et al. Hippocampal saltinducible kinase 2 plays a role in depression via the CREB-regulated transcription coactivator 1-CAMP response element binding-brain-derived neurotrophic factor pathway. Biol. Psychiatry. 2019;85:650-66.

16. Malm HA, Mollet IG, Berggreen C, Orho-Melander M, Esguerra JL, Goransson O, et al. Transcriptional regulation of the miR-212/miR-132 cluster in insulinsecreting beta-cells by CAMP-regulated transcriptional co-activator 1 and saltinducible kinases. Mol Cell Endocrinol. 2016;424:23-33.

17. Obermeier B, Daneman R, Ransohoff RM. Development, maintenance and disruption of the blood-brain barrier. Nat Med. 2013;19:1584-96.

18. Jiang X, Andjelkovic AV, Zhu L, Yang T, Bennett MVL, Chen J, et al. Blood-brain barrier dysfunction and recovery after ischemic stroke. Prog Neurobiol. 2018;163164:144-71.
19. Liebner S, Dijkhuizen RM, Reiss Y, Plate KH, Agalliu D, Constantin G. Functional morphology of the blood-brain barrier in health and disease. Acta Neuropathol. 2018;135:311-36.

20. Terstappen GC, Meyer AH, Bell RD, Zhang W. Strategies for delivering therapeutics across the blood-brain barrier. Nat Rev Drug Discov. 2021;20:362-383.

21. Greene C, Hanley N, Campbell M. Claudin-5: gatekeeper of neurological function. Fluids Barriers CNS. 2019;16:3.

22. Nitta T, Hata $M$, Gotoh $S$, Seo $Y$, Sasaki $H$, Hashimoto $N$, et al. Size-selective loosening of the blood-brain barrier in claudin-5-deficient mice. J Cell Biol. 2003;161:653-60.

23. Sladojevic N, Stamatovic SM, Johnson AM, Choi J, Hu A, Dithmer S, et al. Claudin1-dependent destabilization of the blood-brain barrier in chronic stroke. J Neurosci. 2019;39:743-57.

24. Balkaya M, Krober JM, Rex A, Endres M. Assessing post-stroke behavior in mouse models of focal ischemia. J Cereb Blood Flow Metab. 2013;33:330-8.

25. Xu B, Zhang Y, Du XF, Li J, Zi HX, Bu JW, et al. Neurons secrete miR-132-containing exosomes to regulate brain vascular integrity. Cell Res. 2017;27:882-97.

26. El Fatimy R, Li S, Chen Z, Mushannen T, Gongala S, Wei Z, et al. MicroRNA-132 provides neuroprotection for tauopathies via multiple signaling pathways. Acta Neuropathol. 2018;136:537-55.

27. Zhang Y, Han B, He Y, Li D, Ma X, Liu Q, et al. MicroRNA-132 attenuates neurobehavioral and neuropathological changes associated with intracerebral hemorrhage in mice. Neurochem Int. 2017;107:182-90.

28. Kanki H, Sasaki T, Matsumura S, Kawano T, Todo K, Okazaki S, et al. CREB coac tivator CRTC2 plays a crucial role in endothelial function. J Neurosci. 2020;40:9533-46.

29. Mendioroz M, Celarain N, Altuna M, Sanchez-Ruiz de Gordoa J, Zelaya MV, Roldan $M$, et al. CRTC1 gene is differentially methylated in the human hippocampus in Alzheimer's disease. Alzheimers Res Ther. 2016;8:15.

30. Parra-Damas A, Valero J, Chen M, Espana J, Martin E, Ferrer I, et al. Crtc1 activates a transcriptional program deregulated at early Alzheimer's disease-related stages. J Neurosci. 2014;34:5776-87.

31. Espana J, Valero J, Minano-Molina AJ, Masgrau R, Martin E, Guardia-Laguarta C et al. beta-Amyloid disrupts activity-dependent gene transcription required for memory through the CREB coactivator CRTC1. J Neurosci. 2010;30:9402-10.

32. Jeong $H$, Cohen DE, Cui L, Supinski A, Savas JN, Mazzulli JR, et al. Sirt1 mediates neuroprotection from mutant huntingtin by activation of the TORC1 and CREB transcriptional pathway. Nat Med. 2011;18:159-65.

33. Chaturvedi RK, Hennessey T, Johri A, Tiwari SK, Mishra D, Agarwal S, et al. Transducer of regulated CREB-binding proteins (TORCs) transcription and function is impaired in Huntington's disease. Hum Mol Genet. 2012;21:3474-88.

34. Nonaka M, Kim R, Fukushima H, Sasaki K, Suzuki K, Okamura M, et al. Regionspecific activation of CRTC1-CREB signaling mediates long-term fear memory. Neuron. 2014:84:92-106.

35. Gehman LT, Stoilov P, Maguire J, Damianov A, Lin CH, Shiue L, et al. The splicing regulator Rbfox1 (A2BP1) controls neuronal excitation in the mammalian brain. Nat Genet. 2011;43:706-11.

36. Stamatovic SM, Phillips CM, Martinez-Revollar G, Keep RF, Andjelkovic AV. Involvement of epigenetic mechanisms and non-coding rnas in blood-brain barrier and neurovascular unit injury and recovery after stroke. Front Neurosci. 2019;13:864.

37. Tsukita S, Tanaka H, Tamura A. The claudins: from tight junctions to biological systems. Trends Biochem Sci. 2019;44:141-52.

38. Tokumasu R, Yamaga K, Yamazaki Y, Murota H, Suzuki K, Tamura A, et al. Dosedependent role of claudin-1 in vivo in orchestrating features of atopic dermatitis. Proc Natl Acad Sci USA. 2016;113:E4061-8.

39. Burek M, Konig A, Lang M, Fiedler J, Oerter S, Roewer N, et al. Hypoxia-induced microRNA-212/132 alter blood-brain barrier integrity through inhibition of tight junction-associated proteins in human and mouse brain microvascular endothelial cells. Transl Stroke Res. 2019;10:672-83.

40. Wolburg H, Lippoldt A. Tight junctions of the blood-brain barrier. Vasc Pharmacol. 2002;38:323-37.

41. Greene C, Campbell M. Tight junction modulation of the blood brain barrier: CNS delivery of small molecules. Tissue Barriers. 2016;4:e1138017.

42. Tran KA, Zhang X, Predescu D, Huang X, Machado RF, Gothert JR, et al. Endothelial beta-catenin signaling is required for maintaining adult blood-brain barrier integrity and central nervous system homeostasis. Circulation. 2016;133:177-86.

43. Kawabe $H$, Nakanishi $H$, Asada M, Fukuhara A, Morimoto K, Takeuchi M, et al. Pilt, a novel peripheral membrane protein at tight junctions in epithelial cells. J Biol Chem. 2001;276:48350-5.

44. Tamaki H, Sanda M, Katsumata O, Hara Y, Fukaya M, Sakagami H. Pilt is a coiledcoil domain-containing protein that localizes at the trans-Golgi complex and regulates its structure. FEBS Lett. 2012;586:3064-70.

45. Conkright MD, Canettieri G, Screaton R, Guzman E, Miraglia L, Hogenesch JB, et al TORCs: transducers of regulated CREB activity. Mol Cell. 2003;12:413-23. 
46. Screaton RA, Conkright MD, Katoh Y, Best JL, Canettieri G, Jeffries S, et al. The CREB coactivator TORC2 functions as a calcium- and CAMP-sensitive coincidence detector. Cell 2004;119:61-74.

47. Kovacs KA, Steullet $P$, Steinmann M, Do KQ, Magistretti PJ, Halfon O, et al. TORC1 is a calcium- and cAMP-sensitive coincidence detector involved in hippocampal long-term synaptic plasticity. Proc Natl Acad Sci. USA. 2007;104:4700-5.

48. Li S, Zhang C, Takemori H, Zhou Y, Xiong ZQ. TORC1 regulates activity-dependent CREB-target gene transcription and dendritic growth of developing cortical neurons. J Neurosci. 2009;29:2334-43.

49. Ch'ng TH, Uzgil B, Lin P, Avliyakulov NK, O'Dell TJ, Martin KC. Activity-dependent transport of the transcriptional coactivator CRTC1 from synapse to nucleus. Cell. 2012;150:207-21.

50. Altarejos JY, Montminy M. CREB and the CRTC co-activators: sensors for hormonal and metabolic signals. Nat Rev Mol Cell Biol. 2011;12:141-51.

51. Yoshimi K, Kunihiro $Y$, Kaneko T, Nagahora H, Voigt B, Mashimo T. ssODNmediated knock-in with CRISPR-Cas for large genomic regions in zygotes. Nat Commun. 2016;7:10431.

52. Kanki H, Sasaki T, Matsumura S, Yokawa S, Yukami T, Shimamura M, et al. betaarrestin-2 in PAR-1-biased signaling has a crucial role in endothelial function via PDGF-beta in stroke. Cell Death Dis. 2019;10:100.

53. Watanabe A, Sasaki T, Yukami T, Kanki H, Sakaguchi M, Takemori H, et al. Serine racemase inhibition induces nitric oxide-mediated neurovascular protection during cerebral ischemia. Neuroscience 2016;339:139-49.

54. Garcia JH, Wagner S, Liu KF, Hu XJ. Neurological deficit and extent of neuronal necrosis attributable to middle cerebral artery occlusion in rats. Stat Valid Stroke. 1995:26:627-34.

55. Terasaki Y, Sasaki T, Yagita Y, Okazaki S, Sugiyama Y, Oyama N, et al. Activation of NR2A receptors induces ischemic tolerance through CREB signaling. J Cereb Blood Flow Metab. 2010;30:1441-9.

\section{ACKNOWLEDGEMENTS}

This work was supported by the following grants: JSPS KAKENHI Grant number $21 \mathrm{~K} 07414$ (to TS), 19H02909 (to SM), and Smoking Research Foundation (to TS) We thank Prof. Marc Montminy, from Salk Institute for Biologic Studies for helpful discussions. The author Haomin Yan would like to thank China Scholarship Council (CSC) for a scholarship support.

\section{AUTHOR CONTRIBUTIONS}

$\mathrm{HY}, \mathrm{TS}$, and HM planned the research idea and experiment design. HY, HK, and KN accomplished the experiments. TS, SM, and HT performed CRTC1 deletion by Crisper/
Cas9. HY and TS drafted the manuscript. Critical revisions of the manuscript were made by all authors.

\section{COMPETING INTERESTS}

The authors declare no competing interests.

\section{ETHICAL APPROVAL}

The animal experiment protocol was approved by the Institutional Animal Care and Use Committee of Osaka University Graduate School of Medicine.

\section{ADDITIONAL INFORMATION}

Supplementary information The online version contains supplementary material available at https://doi.org/10.1038/s41420-021-00773-w.

Correspondence and requests for materials should be addressed to Tsutomu Sasaki.

Reprints and permission information is available at http://www.nature.com/ reprints

Publisher's note Springer Nature remains neutral with regard to jurisdictional claims in published maps and institutional affiliations.

(i) Open Access This article is licensed under a Creative Commons Attribution 4.0 International License, which permits use, sharing, adaptation, distribution and reproduction in any medium or format, as long as you give appropriate credit to the original author(s) and the source, provide a link to the Creative Commons license, and indicate if changes were made. The images or other third party material in this article are included in the article's Creative Commons license, unless indicated otherwise in a credit line to the material. If material is not included in the article's Creative Commons license and your intended use is not permitted by statutory regulation or exceeds the permitted use, you will need to obtain permission directly from the copyright holder. To view a copy of this license, visit http://creativecommons. org/licenses/by/4.0/.

(c) The Author(s) 2021 